\title{
Lack of endogenous parathyroid hormone delays fracture healing by inhibiting vascular endothelial growth factor-mediated angiogenesis
}

\author{
QINGFENG DING $^{1 *}$, PENG SUN $^{1,2 *}$, HAO ZHOU $^{1}$, BOWEN WAN $^{1,3}$, JIAN YIN $^{1}$, \\ YAO HUANG ${ }^{1}$, QINGQING LI ${ }^{1}$, GUOYONG YIN ${ }^{1}$ and JIN FAN ${ }^{1}$ \\ ${ }^{1}$ Orthopaedic Department, The First Affiliated Hospital of Nanjing Medical University, Nanjing, Jiangsu 210029; \\ ${ }^{2}$ Orthopaedic Department, Huai'an First People's Hospital, Huai'an, Jiangsu 223300; ${ }^{3}$ Orthopaedic Department, \\ The Affiliated Zhengjiang First People's Hospital, Zhengjiang, Jiangsu 212000, P.R. China
}

Received March 3, 2016; Accepted December 19, 2017

DOI: $10.3892 /$ ijmm.2018.3614

\begin{abstract}
Intermittent low-dose injections of parathyroid hormone (PTH) have been reported to exert bone anabolic effects and to promote fracture healing. As an important proangiogenic cytokine, vascular endothelial growth factor (VEGF) is secreted by bone marrow mesenchymal stem cells (BMSCs) and osteoblasts, and serves a crucial regulatory role in the process of vascular development and regeneration. To investigate whether lack of endogenous PTH causes reduced angiogenic capacity and thereby delays the process of fracture healing by downregulating the VEGF signaling pathway, a PTH knockout (PTHKO) mouse fracture model was generated. Fracture healing was observed using X-ray and micro-computerized tomography. Bone anabolic and angiogenic markers were analyzed by immunohistochemistry and western blot analysis. The expression levels of VEGF and associated signaling pathways in murine BMSC-derived osteoblasts were measured by quantitative polymerase chain reaction and western blot analysis. The expression levels of protein kinase A (PKA), phosphorylated-serine/threonine protein kinase (pAKT), hypoxia-inducible factor-1 $\alpha$ (HIF1 $\alpha)$ and VEGF were significantly decreased in BMSC-derived osteoblasts from PTHKO mice. In addition, positive platelet endothelial cell adhesion molecule staining was reduced in PTHKO mice, as determined by immunohistochemistry. The expression levels of HIF1 $\alpha$, VEGF, runt-related transcription
\end{abstract}

Correspondence to: Dr Guoyong Yin, Orthopaedic Department, The First Affiliated Hospital of Nanjing Medical University, 300 Guangzhou Road, Nanjing, Jiangsu 210029, P.R. China

E-mail: guoyong_yin@sina.com

*Contributed equally

Key words: parathyroid hormone, protein kinase A, phosphorylated-serine/threonine protein kinase, hypoxia-inducible factor- $1 \alpha$, vascular endothelial growth factor, bone healing factor 2, osteocalcin and alkaline phosphatase were also decreased in PTHKO mice, and fracture healing was delayed. In conclusion, lack of endogenous PTH may reduce VEGF expression in BMSC-derived osteoblasts by downregulating the activity of the PKA/pAKT/HIF1 $\alpha / \mathrm{VEGF}$ pathway, thus affecting endochondral bone formation by causing a reduction in angiogenesis and osteogenesis, ultimately leading to delayed fracture healing.

\section{Introduction}

Fracture repair progresses through the following stages: Hematoma formation, fibrocartilaginous callus formation, bony callus formation and bone remodeling $(1,2)$. Fractures are often accompanied by ruptured blood vessels, thus causing local hypoxia. In a hypoxic microenvironment, bone marrow mesenchymal stem cells (BMSCs) differentiate into chondrocytes and form an area of cartilage in the central region of the fracture. Mature cartilage cells can secrete a large amount of cartilage matrix factors, and new blood vessels then grow into the cartilaginous callus along the matrix. Revascularization improves the local oxygen supply, stimulating osteoblastic differentiation of BMSCs and bone-lining cells in coordination with bone morphogenetic proteins (BMPs), runt-related transcription factor 2 (RUNX2) and other cytokines, thus promoting endochondral bone formation and matrix calcification, which subsequently form the bony callus and ultimately lead to fracture healing (3-5).

Tissue hypoxia can induce angiogenesis following fracture, and hypoxia-inducible factor (HIF) is the key protein expressed in most tissues in response to localized low oxygen tension (6). Under normoxic conditions, HIF1 $\alpha$ binds to the von Hippel-Lindau tumor suppressor (pVHL) and is subsequently hydrolyzed. Conversely, this process is suppressed under hypoxic conditions, and HIF1 $\alpha$ forms dimers with HIF1 $\beta$, which then enter the nucleus and activate transcription of HIF target genes (7-9). Vascular endothelial growth factor (VEGF) is the most important HIF target gene $(10,11)$. A significant reduction in the formation of blood vessels and bone is observed in vascular endothelial-cell-specific HIF1 $\alpha$-deficient 
mice. Significantly increased metaphyseal vascular volume and bone formation have been observed in pVHL knockout mice, whereas administration of a VEGF receptor antagonist can offset angiogenesis caused by excessive activation of HIF1 $\alpha$ (10). Reduced bone density, as well as decreased amounts of trabecular bone and thinner cortical bone have been detected in VEGF knockout mice. In vitro studies have demonstrated that osteoblasts in VEGF knockout mice exhibit diminished capacity for differentiation and maturation (12).

Parathyroid hormone (PTH) is an 84-amino acid peptide hormone secreted by the parathyroid gland. Numerous studies have demonstrated that intermittent low-dose injections of recombinant human (rh)PTH can increase bone anabolism and promote fracture healing $(13,14)$. Conversely, trabecular bone volume and osteoblast number are significantly reduced $(15,16)$, and fracture healing is delayed in PTH knockout (PTHKO) mice (17). At present, the mechanisms by which PTH promotes fracture healing remain to be elucidated; stimulation of angiogenesis may be one of the mechanisms (18-20). A previous study indicated that the effects of PTH on osteogenesis are predominantly mediated by adenylate cyclase (AC)/cyclic adenosine monophosphate (cAMP)/protein kinase A (PKA)/cAMP response element-binding protein pathway (21). In addition, it has been confirmed that the activated $\mathrm{cAMP} / \mathrm{PKA} /$ phosphorylated-serine/threonine protein kinase $(\mathrm{pAKT}) / \mathrm{HIF} 1 \alpha / \mathrm{VEGF}$ signaling pathway can promote angiogenesis (22). Previous studies have revealed that PTH can quickly promote the expression of VEGF in osteoblasts $(13,23)$. Therefore, the present study hypothesized that PTH may affect fracture healing through its effects on angiogenesis mediated by the PKA/pAKT/HIF1 $\alpha /$ VEGF pathway. In the present study, a PTHKO mouse fracture model was constructed, and fracture healing and the expression of associated angiogenic factors were measured, in order to investigate whether endogenous PTH affects angiogenesis via the PKA/pAKT/ HIF1 $\alpha /$ VEGF pathway, thus stimulating fracture healing.

\section{Materials and methods}

Reagents. Anti-mouse platelet endothelial cell adhesion molecule (PECAM; 3528; 1:100) and GAPDH (5174/51332) were purchased from Cell Signaling Technology, Inc. (Danvers, MA, USA) and osteocalcin (OCN; ab93876; 1:400) monoclonal antibodies were purchased from Abcam (Cambridge, MA, USA). RUNX2 (ab76956; 1:600), HIF1 $\alpha$ (ab8366; 1:600), pVEGF receptor 2 (pVEGFR2; ab131241; 1:200), proliferating cell nuclear antigen (PCNA; ab92552; 1:400), VEGF (ab46154; 1:200), PKA (ab75991) and pAKT monoclonal antibodies (ab81283) were purchased from Abcam (Cambridge, MA, USA). Type II collagen (COL II; sc-7763; 1:200) primary antibody and all secondary antibodies were purchased from Santa Cruz Biotechnology, Inc. (Dallas, TX, USA). Streptavidin-biotin complex (SABC) immunohistochemistry kit and DAB chromogenic kit were purchased from Vector Laboratories, Inc. (Burlingame, CA, USA). Polymerase chain reaction (PCR) kit (PCR kit Takara A5003) was provided by Takara Bio, Inc. (Otsu, Japan). High glucose or low glucose Dulbecco's modified Eagle's medium (DMEM), and fetal bovine serum were purchased from HyClone; GE Healthcare (Logan, UT, USA). Penicillin-streptomycin double-resistant solution was purchased from Gibco; Thermo Fisher Scientific, Inc. (Waltham, MA, USA). Dexamethasone, $\beta$-glycerophosphate and rhPTH (1-34) were purchased from Sigma-Aldrich; Merck KGaA (Darmstadt, Germany). PBS and total protein extraction kit [containing radioimmunoprecipitation assay (RIPA) buffer and phenylmethylsulfonyl fluoride) were provided by Beyotime Institute of Biotechnology (Nantong, China). mRNA extraction kit was purchased from Qiagen GmbH (Hilden, Germany). Alkaline phosphatase (ALP) staining kit was provided by Nanjing Jiancheng Bioengineering Institute (Nanjing, China). Osteogenic induction medium was prepared by adding dexamethasone, ascorbic acid and $\beta$-glycerophosphate to basal medium and serum at final concentrations of $10 \mathrm{nmol} / 1,50 \mu \mathrm{g} / \mathrm{ml}$ and $10 \mathrm{mmol} / \mathrm{l}$, respectively.

Construction and identification of PTHKO mice. Briefly, the PTHKO mouse model was constructed by inserting a neomycin resistance gene (NEO) into exon III of the mouse PTH gene to replace the entire mature PTH coding sequence (24). Parental knockout mice were provided by McGill University (Montreal, QC, Canada), and were housed in a specific pathogen-free (SPF) standard animal facility under the following conditions: Humidity, 45-75\%; temperature, $22-26^{\circ} \mathrm{C}$; $12 \mathrm{~h}$ light/12 h dark cycle, in the specific-pathogen-free animal facility at the Experimental Animal Center of Nanjing Medical University (Nanjing, China); with ad libitum access to food and water. Mouse genotypes and PTH gene knockout were confirmed by PCR amplification of DNA fragments. Primer sequences were as follows: PTH, forward 5'-GATGTGTGCAAACA CCGTGGCTAA-3', reverse 5'-TCCAAAGTTTCATTA CAGTAGAAG-3'; and NEO, forward 5'-TCTTGATTCCCA CTTTGTGGTTCTA-3', reverse primer sequence was the same as the PTH forward primer. Annealing temperature was $55^{\circ} \mathrm{C}$. For experiments, 18 two-month-old PTHKO mice and 15 wild-type (WT) littermates were randomly selected regardless of gender. Their weigh was 20-30 g, WT were heavier than the PTHKO mice. The present study was approved by the Ethics Committee of Nanjing Medical University.

Establishment and analysis of the murine fracture model. The murine fracture model was established as follows: Mice were anesthetized with an intraperitoneal injection of ketamine/xylazine. Subsequently, skin tissue around the knee was disinfected and a $25 \mathrm{G}$ needle was inserted through the patellar tendon into the femoral canal. A femoral fracture was created by 3-point bending using an Einhorn device, and was confirmed by X-ray examination (Faxitron Bioptics, LLC, Tucson, AZ, USA). Each mouse received daily subcutaneous injections of $0.5 \mathrm{mg} / \mathrm{kg}$ buprenorphine for 3 consecutive days (25). Fracture healing was assessed by X-ray radiography and callus formation was examined using Skyscan 1176 high-resolution micro-computerized tomography (micro-CT) (Bruker Corporation, Billerica, MA, USA) at 1 and 2 weeks after establishment of the fracture model.

Cell culture. For BMSCs culture, mice were sacrificed and immediately immersed in $75 \%$ alcohol for 10 min. Bilateral femurs were then removed under sterile conditions, and the marrow cavity was repeatedly flushed using serum-free low glucose DMEM to obtain a single-cell suspension. Cells were 
A



B

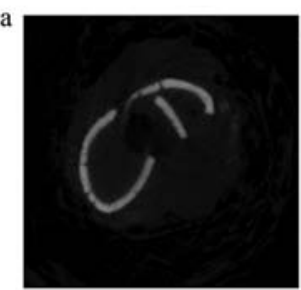

1 Week
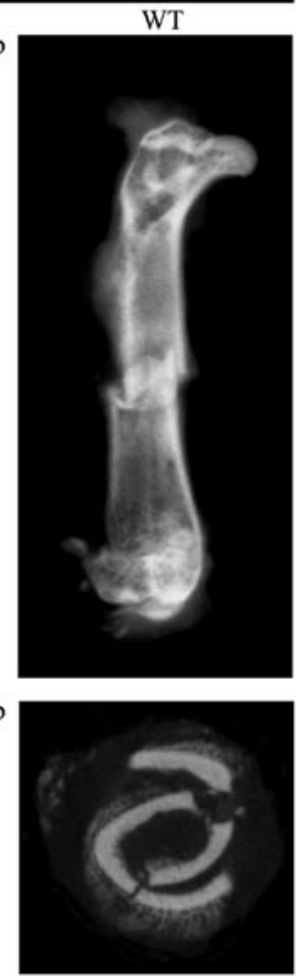

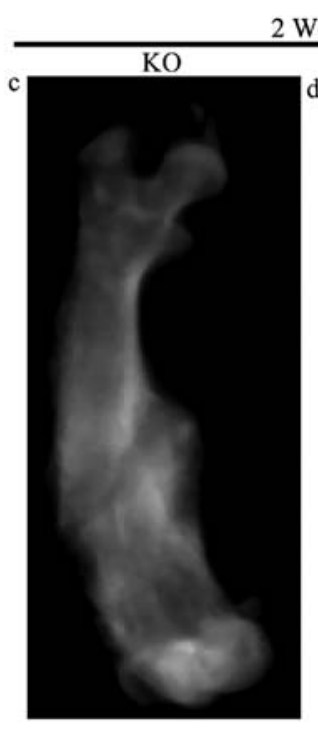

2 Week
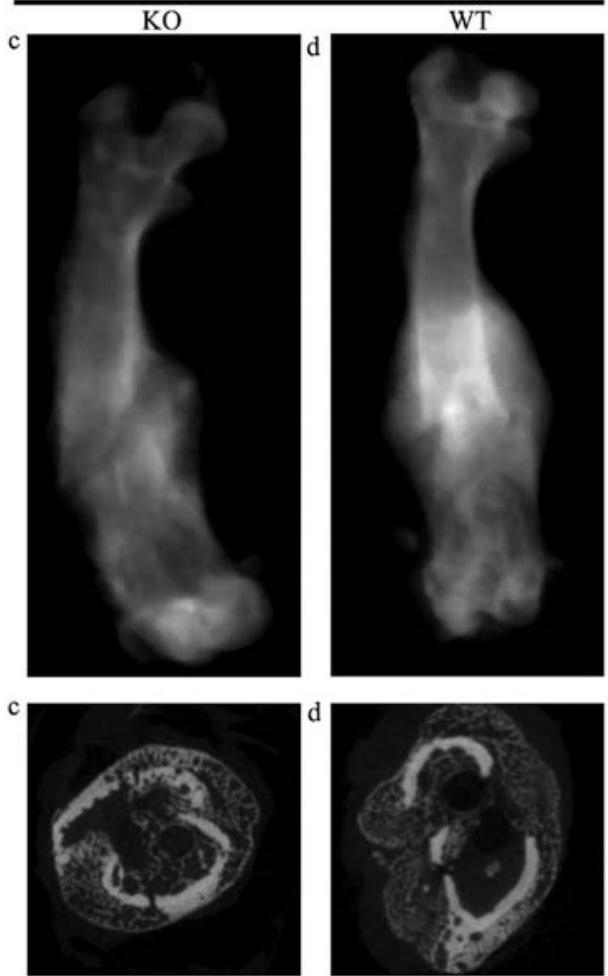

Figure 1. (A) X-ray images of fractures in (a) PTHKO and (b) PTHWT mice 1 week after fracture. Although fracture lines were clearly visible and a small region of calcification was observed in the surrounding area of fractures in both groups, the calcified region in PTHKO mice was much smaller than that in WT mice. (c) Fracture line remained clear and exogenous callus was relatively small in PTHKO mice 2 weeks after fracture. (d) Blurry fracture line was observed, and the exogenous callus area was clear and relatively large in PTHWT mice 2 weeks after fracture. (B) Coronal micro-computerized tomography views of fractures in (a) PTHKO and (b) PTHWT mice at 1 week and in (c) PTHKO and (d) PTHWT mice at 2 weeks after fracture. PTH, parathyroid hormone; PTHKO, PTH knockout; PTHWT, PTH wild-type.

collected by centrifugation at $1,200 \mathrm{xg} / \mathrm{min}$ for $5 \mathrm{~min}$, then resuspended, inoculated into culture flasks, and incubated in a humidified incubator in an atmosphere containing $5 \% \mathrm{CO}_{2}$ at $37^{\circ} \mathrm{C}$. The $3 \mathrm{rd}$ generation of BMSCs was induced to differentiate into osteoblasts. Briefly, BMSCs from WT (+/+) and PTHKO (-/-) mice $\left(5 \times 10^{6} / \mathrm{ml}\right)$ were inoculated into 6 -well plates and into culture plates containing slides; induction medium containing exogenous PTH (final concentration, $10^{-7} \mathrm{~mol} / \mathrm{l}$ ) was added to the cells once they reached $80 \%$ confluence. Induction medium was replaced with ordinary culture medium after $6 \mathrm{~h}$. The cells were treated with PTH for $6 \mathrm{~h}$ every $48 \mathrm{~h}$. Cells were collected 1 and 2 weeks after induction, and protein and RNA were extracted using specific kits according to the manufacturers' protocols. ALP staining of fixed cells was performed using an ALP staining kit according to the manufacturer's protocol.

Coculture of human umbilical vein endothelial cells (HUVECs) and BMSCs. BMSCs were cultured as aforementioned (for 2 weeks) and allowed to reach $80 \%$ confluence, after which they were treated with induction medium, and HUVECS were cocultured with the lower layer of BMSCs for 1 week. Dishes were observed on day 7 after the start of coculture. Cell nuclei were stained with hematoxylin and images were captured.

Immunohistochemical examination. Fracture calluses (captured 1 and 2 weeks after femur fracture) were fixed in
$10 \%$ formalin for $12-24 \mathrm{~h}$ at room temperature $\left(20-25^{\circ} \mathrm{C}\right)$, decalcified in 5\% EDTA-2Na for 4-5 weeks, dehydrated through a graded ethanol series, cleared in xylene, embedded in paraffin wax and cut into $5-\mu \mathrm{m}$ sections. Histological observations were performed after hematoxylin and eosin (H\&E) staining, cartilage morphology was observed using alcian blue staining, and differences in osteoblasts were examined by ALP staining. Immunohistochemical staining, followed by SABC and DAB color development were conducted according to the manufacturer's protocols. Images of sections were captured using a light microscope; four fields of each section were randomly selected, and the positive expression rate and gray values of stained areas were measured under the same light intensity to semi-quantify protein expression levels using Image-Pro-Plus 6.0 (Media Cybernetics, Inc., Rockville, MD, USA).

Western blot analysis. Cells were lysed with RIPA lysis buffer, total protein was extracted and concentration was measured using the Bradford method. Proteins $(10 \mu \mathrm{g})$ were separated by $12 \%$ SDS-PAGE. Following SDS-PAGE, protein samples were transferred to polyvinylidene fluoride (PVDF) membranes (EMD Millipore, Billerica, MA, USA). PVDF membranes were then incubated with primary antibodies (OCN, $1 \mu \mathrm{g} / \mathrm{ml}$; RUNX2, $2 \mu \mathrm{g} / \mathrm{ml}$; VEGF, $1 \mu \mathrm{g} / \mathrm{ml}$; pVEGFR2, $1 \mu \mathrm{g} / \mathrm{ml}$; HIF $1 \alpha, 1 \mu \mathrm{g} / \mathrm{mL}$; PKA, $0.5 \mu \mathrm{g} / \mathrm{ml}$; AKT, $1 \mu \mathrm{g} / \mathrm{ml}$; pAKT, $1 \mu \mathrm{g} / \mathrm{ml}$ ) overnight at $4^{\circ} \mathrm{C}$, followed by incubation with 


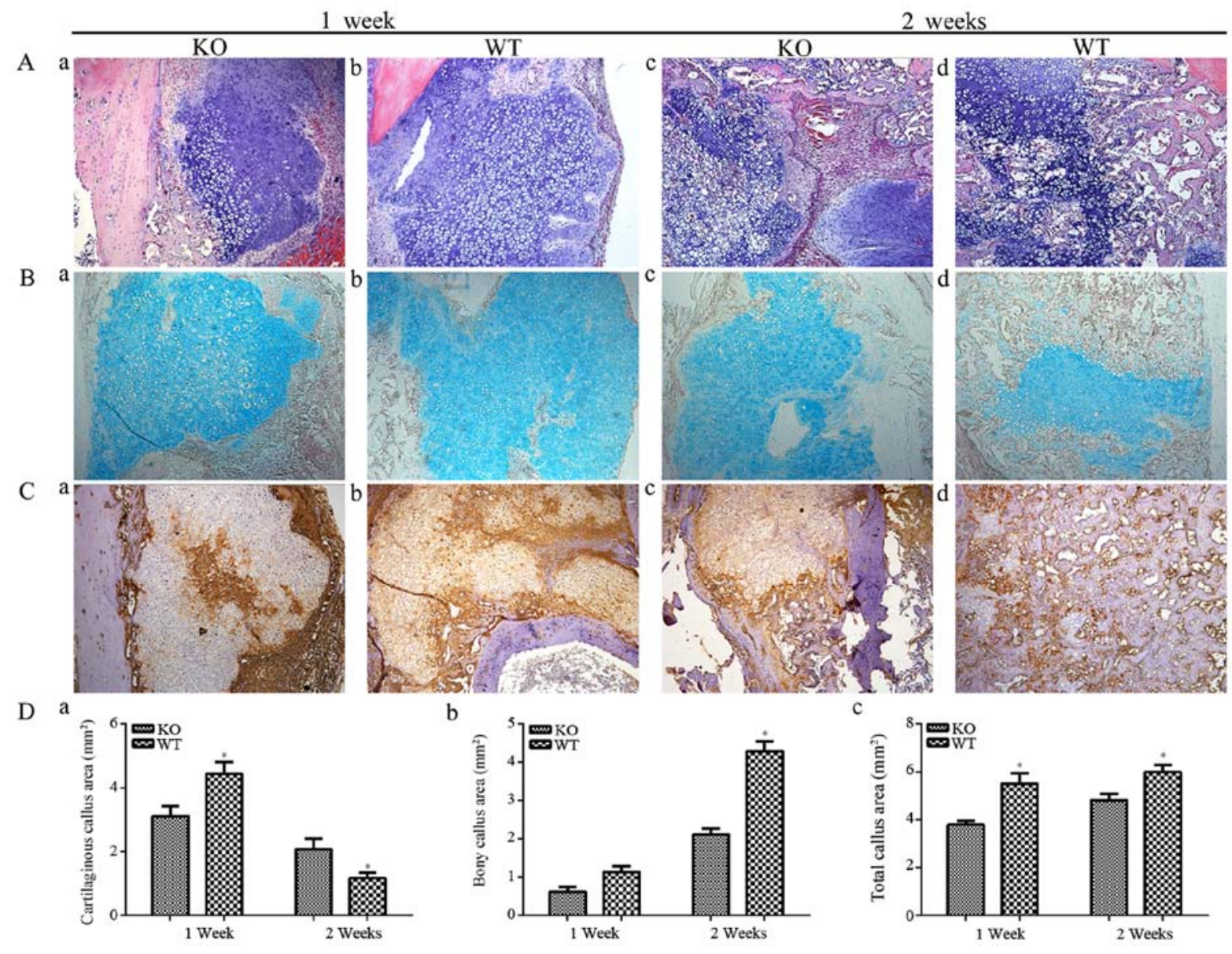

Figure 2. (A) H\&E staining (magnification, x100) of fractures. (a) In PTHKO mice, fractures developed a smaller cartilaginous callus compared with in (b) WT mice 1 week after fracture. (c) In PTHKO mice at 2 weeks after fracture, little endochondral bone formation and a large cartilage area were detected. (d) In WT mice at 2 weeks after fracture, normal endochondral bone formation and decreased cartilage area were detected. (B) Alcian blue staining (magnification, x100) of fractures. Light blue area shows cartilaginous callus, which was consistent with the results of H\&E staining. (C) Immunohistochemical staining of COL II in fractures (magnification, x100) in (a) PTHKO and (b) WT mice 1 week after fracture. WT mice exhibited strong positive staining for COL II; however, COL II expression was detected only in the central region of the cartilage expansion area in PTHKO mice. Immunohistochemical staining of COL II in (c) PTHKO and (d) WT mice 2 weeks after fracture. COL II expression was slightly increased in PTHKO mice, but remained lower than that in WT mice. (D) Statistical analysis of (a) cartilaginous callus area, (b) bony callus area and (c) total callus area. ${ }^{*} \mathrm{P}<0.05$. PTH, parathroid hormone; PTHKO, PTH knockout; WT, wild-type.

horseradish peroxidase-conjugated secondary antibody for $2 \mathrm{~h}$ at $37^{\circ} \mathrm{C}$. Enhanced chemiluminescence (Beyotime Institute of Biotechnology) was used to visualize the bands, and blots were analyzed using Quantity One software (version 4.62; Bio-Rad Laboratories Inc., Hercules, CA, USA).

Reverse transcription-quantitative ( $R T-q P C R$ ). Total RNA was extracted from adherent cells using a kit according to the manufacturer's protocol. Concentration and purity of mRNA were determined using an ultraviolet spectrometer. RT was performed using the Superscript ${ }^{\mathrm{TM}}$ first-strand synthesis system (Invitrogen; Thermo Fisher Scientific, Inc.) in RT reaction buffer (Takara Bio, Inc.) according to Takara PCR kit protocol. The resulting cDNA was quantified using a dual hybridization probe system (forward primer, 5-GATGTCTGCAAACACCGTGGCTAA-3, reverse primer, 5-TCCAAAGTTTCATTACAGTAGAAG-3; Neo, 5-TCTTGATTCCCACTTTGTGGTTCTA-3; Roche Diagnostics) and a semi-qPCR procedure according to the manufacturer's protocol (Takara Bio, Inc.). The PCR conditions were as follows: 1 cycle at $95^{\circ} \mathrm{C}$ for $4 \mathrm{~min}, 30$ cycles at $95^{\circ} \mathrm{C}$ for $30 \mathrm{sec}, 30$ cycles at $55^{\circ} \mathrm{C}$ for $30 \mathrm{sec}, 30$ cycles at $72^{\circ} \mathrm{C}$ for
$30 \mathrm{sec}, 1 \mathrm{cycle}$ at $72^{\circ} \mathrm{C}$ for $10 \mathrm{~min}$ and then 1 cycle at $4^{\circ} \mathrm{C}$. The thermocycling conditions were as follows: degeneration at $95^{\circ} \mathrm{C}$ for $4 \mathrm{~min}$; extension at $72^{\circ} \mathrm{C}$; renaturation at $55^{\circ} \mathrm{C}$.

Statistical analysis. All experimental data are presented as the means \pm standard deviation, and were processed using SPSS 13.0 software (SPSS, Inc., Chicago, IL, USA). All experiments were repeated independently three times. Differences among groups were analyzed by independent-samples t-test or single-factor analysis of variance. IHC data were analysed by ANOVA followed by Boferroni's post hoc test. $\mathrm{P}<0.05$ was considered to indicate a statistically significant difference (26).

\section{Results}

Delayed fracture healing in PTHKO mice. X-ray examination demonstrated that fracture lines were clearly visible and a small region of calcification was observed in the surrounding area close to fractures in WT and PTHKO mice 1 week after fracture. However, the calcified region in the PTHKO mice was smaller than that in the WT mice (Fig. 1Aa and b). 

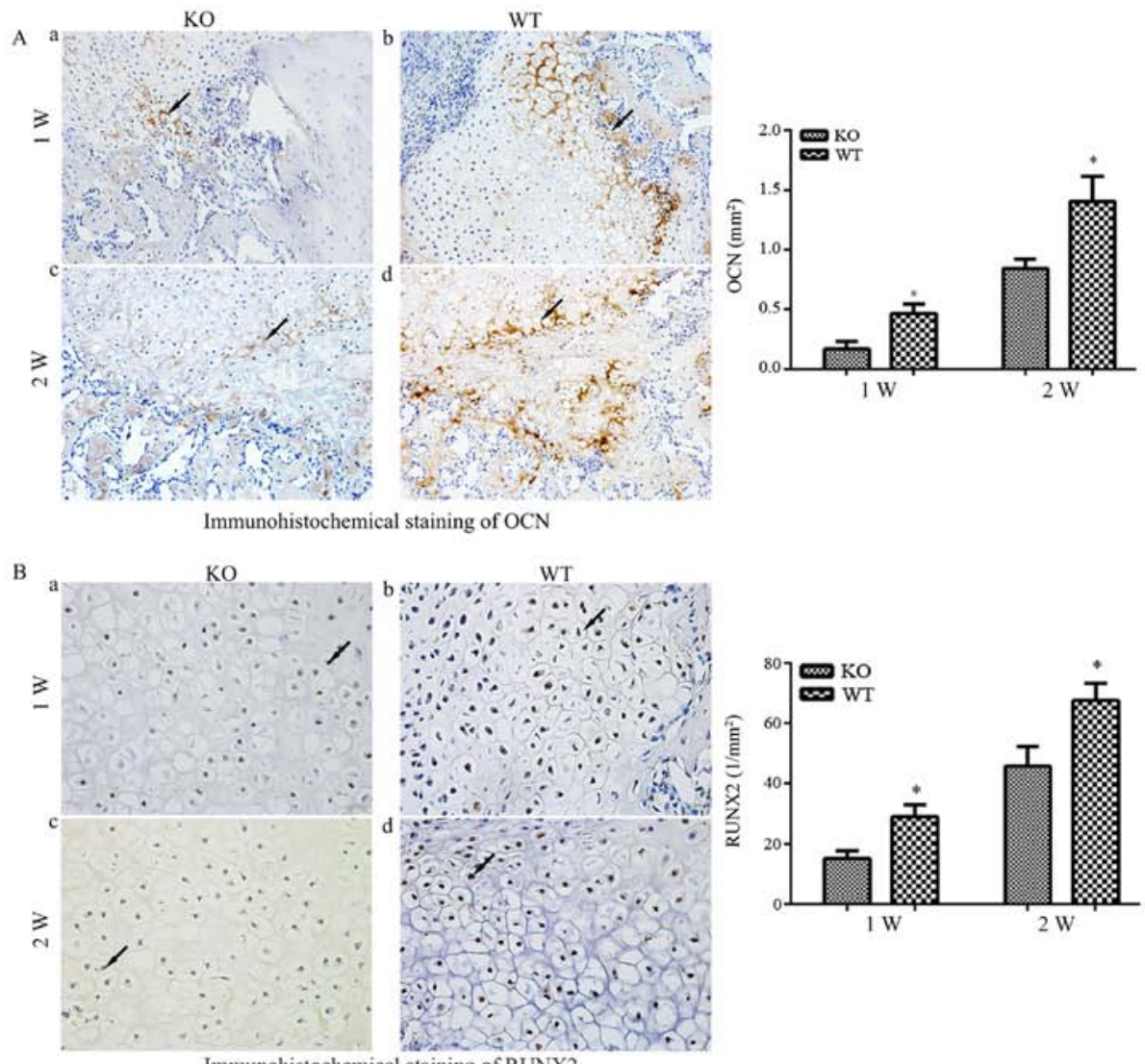
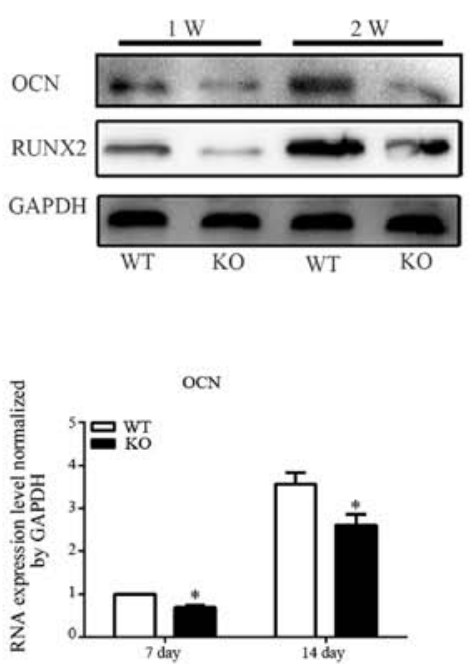
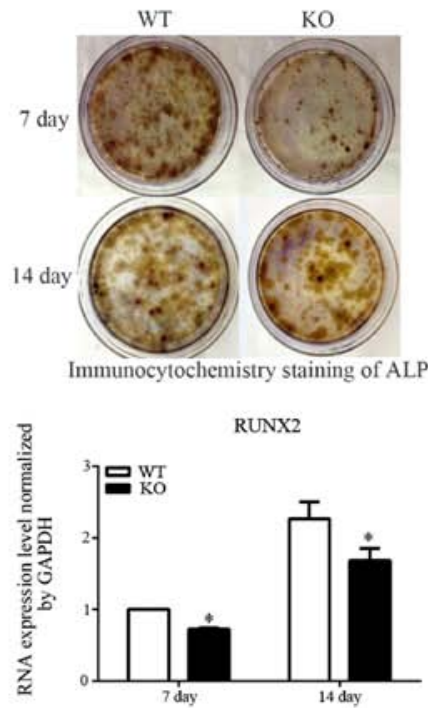

Figure 3. (A) Immunohistochemical staining of OCN (magnification, x200) in fractures of (a) PTHKO and (b) WT mice 1 week after fracture. OCN expression was significantly lower in PTHKO mice compared with in WT mice and was primarily distributed around the edge of the callus. Immunohistochemical staining of OCN in fractures of (c) PTHKO and (d) WT mice 2 weeks after fracture. OCN expression was significantly reduced in PTHKO mice compared with in WT mice; however, it was increased in both groups compared with after 1 week. (B) Immunohistochemical staining of RUNX2 (magnification, $\mathrm{x} 400$ ) in fractures of (a) PTHKO and (b) WT mice 1 week after fracture. RUNX2 expression was significantly lower in PTHKO mice compared with in WT mice, although it was relatively strong in the area surrounding the cartilaginous callus in both groups. Immunohistochemical staining of RUNX2 in fractures of (c) PTHKO and (d) WT mice 2 weeks after fracture. RUNX2 was strongly expressed in WT mice, whereas RUNX2 expression remained lower in PTHKO mice compared with in WT mice, and was mainly concentrated in the outer periphery of the cartilaginous callus. Black arrows indicate positive areas. (C) Callus proteins, including OCN and RUNX2, were detected using western blot analysis. (D) ALP immunocytochemistry. Reduced ALP staining intensity was detected in PTHKO mice compared with in WT mice. (E) Reverse transcription-quantiative polymrease chain reaction analysis of mRNA expression of (a) OCN and (b) RUNX2 in BMSC-derived osteoblasts; the mRNA expression levels were significantly lower in PTHKO mice compared with in WT mice 1 and 2 weeks after induction. ${ }^{*} \mathrm{P}<0.05$. ALP, alkaline phosphatase; BMSC, bone marrow mesenchymal stem cells; OCN, osteocalcin; PTH, parathroid hormone; PTHKO, PTH knockout; RUNX2, runt-related transcription factor 2; WT, wild-type.

Furthermore, fracture lines were less distinct in WT mice 2 weeks after fracture, but remained clearly visible in
PTHKO mice. PTHKO mice exhibited relatively smaller calluses compared with WT mice (Fig. 1Ac and d). 

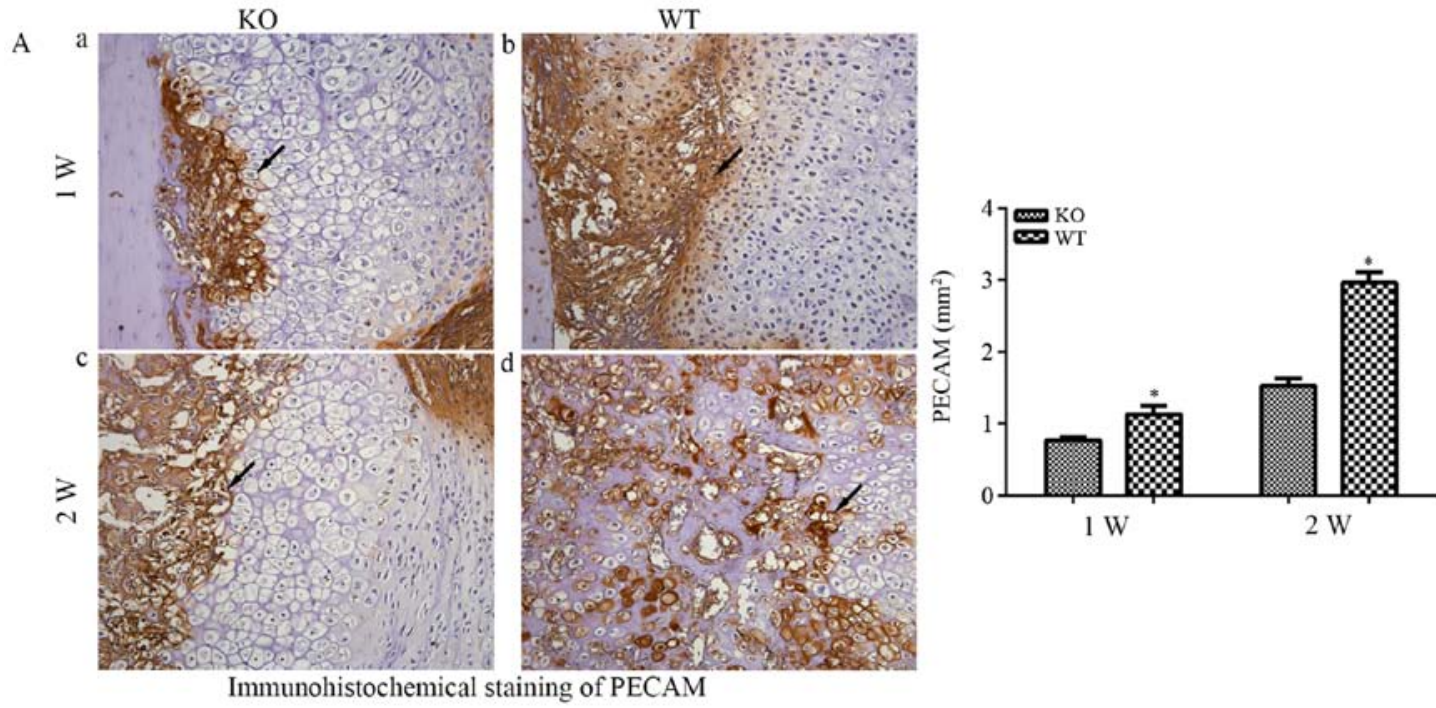

B

$\mathrm{KO}$

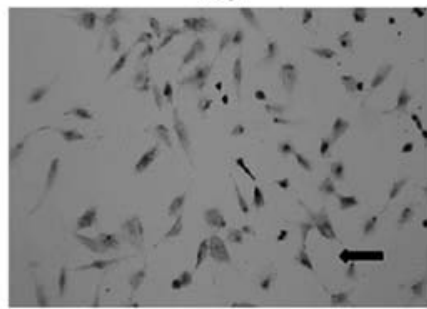

WT

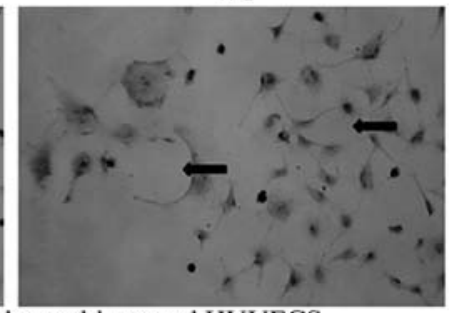

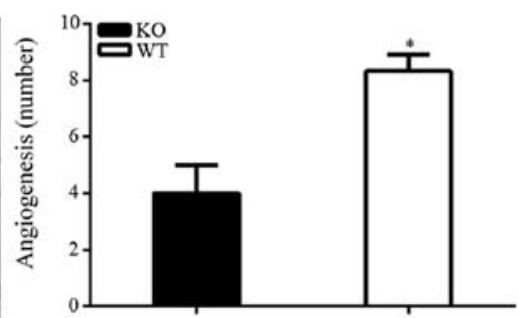

Figure 4. (A) Immunohistochemical staining of PECAM (magnification, x200) in fractures. (a) In PTHKO and (b) WT mice 1 week after fracture, the area of angiogenesis was significantly smaller in PTHKO mice compared with in WT mice. (c) In PTHKO and (d) WT mice 2 weeks after fracture, a large number of blood vessels were observed in the cartilaginous callus in WT mice, whereas fewer blood vessels were detected in PTHKO mice and were mainly located abound the cartilaginous callus. Black arrows indicate positive areas. (B) BMSCs-derived osteoblasts were cocultured with HUVECs for 2 weeks. HUVECs cocultured with PTHKO BMSCs-derived osteoblasts displayed a significantly reduced capacity for aggregation and cross-linking. Black arrows indicate cross-linked HUVECs. ${ }^{*}$ P $<0.05$. BMSC, bone marrow mesenchymal stem cells; HUVECs, human umbilical vein endothelial cells; PECAM, platelet endothelial cell adhesion molecule; PTH, parathroid hormone; PTHKO, PTH knockout; WT, wild-type.

Coronal micro-CT views and three-dimensional surface reconstruction analysis of fracture lines revealed no differences between bone volume (BV), total volume (TV), bone surface (BS), BS/TV and trabecular number (Tb.N) in PTHKO mice compared with WT mice at 1 week after fracture. However, PTHKO mice had significantly lower $\mathrm{BV} / \mathrm{TV}$ ratio, reduced trabecular thickness ( $\mathrm{Tb}$.Th), larger trabecular spacing (Tb.Sp) and increased porosity compared with WT mice (Fig. 1Ba and b). A total of 2 weeks after fracture, PTHKO mice exhibited significantly lower BV, TV, BV/TV, BS/TV, Tb.N and Tb.Th, and substantially increased Tb.Sp and porosity compared with in WT mice, thus indicating that PTHKO mice displayed reduced ossification, poor trabecular development and cancellous bone during fracture healing (Fig. 1Bc and d).

H\&E staining of tissue sections demonstrated that PTHKO mice developed a significantly smaller cartilaginous callus compared with WT mice (Fig. 2Aa and b); even though callus formation was detected in both groups 1 week after fracture. WT mice exhibited normal endochondral bone formation 2 weeks after fracture (Fig. 2Ad). Osteoid was formed in and surrounding the cartilage expansion area and cartilage area was reduced (Fig. 2Ad). Conversely, endochondral bone formation was observed only in regions adjacent to the cartilage expansion area in PTHKO mice; chondrocytes were arranged in an abnormal manner, and cartilage areas were significantly larger than those in WT mice (Fig. 2Ac). The results of cartilage-specific alcian blue staining were similar to those detected by $\mathrm{H} \& \mathrm{E}$ staining, but cartilaginous callus is more significant (Fig. 2B). COL II is secreted by chondrocytes and is an important component of the cartilage matrix (27). Immunohistochemical staining revealed that PTHKO mice exhibited impaired ability to secrete COL II in addition to a diminished capacity for endochondral bone formation. WT mice exhibited strong positive staining for COL II 1 week after fracture, whereas COL II expression was identified only in the central region of the cartilage expansion area in $\mathrm{PTHKO}$ mice (Fig. 2Ca and b). Although the expression levels of COL II were slightly increased in PTHKO mice, it remained lower than in WT mice 2 weeks after fracture (Fig. 2Cc and d).

Defective bone formation in PTHKO mice. Expression of $\mathrm{OCN}$, an indicator of osteoblast activity, was examined to further confirm alterations in endochondral bone formation in PTHKO mice (Fig. 3A). Immunohistochemical staining detected higher levels of OCN expression in WT mice 1 week after fracture (Fig. 3Ab), whereas OCN expression was much lower and was observed primarily around the edge of the callus in PTHKO mice (Fig. 3Aa). In addition, PTHKO mice exhibited significantly lower OCN expression compared with in WT 


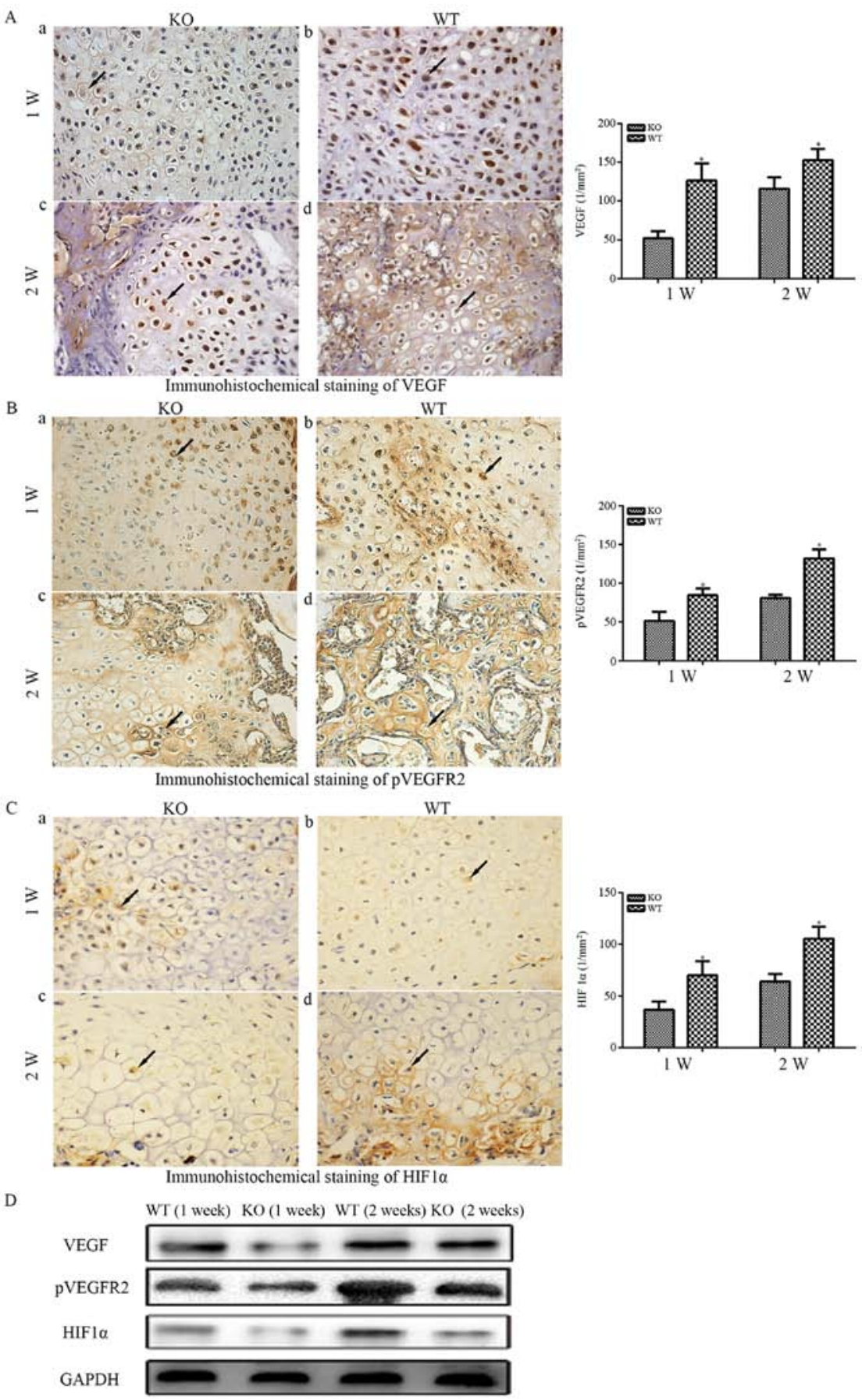

Figure 5. (A) Immunohistochemical staining of VEGF-A (magnification, x400) in fractures. (a) In PTHKO and (b) WT mice 1 week after fracture, VEGF-A expression was significantly reduced in PTHKO mice compared with in WT mice. (c) In PTHKO and (d) WT mice 2 weeks after fracture, VEGF-A expression was increased in PTHKO mice, but remained significantly lower than that in WT mice. (B) Immunohistochemical staining of pVEGFR2 (magnification, x400) in fractures. (a) In PTHKO and (b) WT mice 1 week after fracture, a significantly smaller number of pVEGFR2-positive cells was detected in the cartilaginous callus in PTHKO mice compared with in WT mice. (c) In PTHKO and (d) WT mice 2 weeks after fracture, a large number of pVEGFR2-positive cells was observed in the cartilaginous callus in WT mice, whereas a much lower level of angiogenesis was detected in PTHKO mice. (C) Immunohistochemical staining for HIF1 $\alpha$ (magnification, x400) in fractures. (a) In PTHKO and (b) WT mice 1 week after fracture, the expression levels of cytoplasmic HIF1 $\alpha$ were significantly lower in PTHKO mice. (c) In PTHKO and (d) WT mice 2 weeks after fracture, HIF1 $\alpha$ expression was increased in both groups; however, the expression remained lower in PTHKO mice compared with in WT mice. Black arrows indicate positive areas. (D) Protein expression levels of VEGF, pVEGFR2 and HIF1 $\alpha$ were detected by western blot analysis. HIF1 $\alpha$, hypoxia inducible factor-1 $\alpha$; PTH, parathroid hormone; PTHKO, PTH knockout; pVEGFR, phosphorylated-VEGF receptor 2; VEGF, vascular endothelial growth factor; WT, wild-type.

mice at 2 weeks; however, expression levels were increased in WT and PTHKO mice 2 weeks after fracture (Fig. 3Ac and d).

RUNX2 is an important regulatory factor that promotes bone formation (28). Immunohistochemical examination detected relatively strong RUNX2 expression in the area surrounding the cartilage callus 1 week after fracture; however, expression was significantly reduced in PTHKO mice compared with in WT mice (Fig. 3Ba and b). WT mice exhibited strong positive staining for RUNX2 2 weeks after fracture, whereas RUNX2 expression remained lower in PTHKO mice compared with 


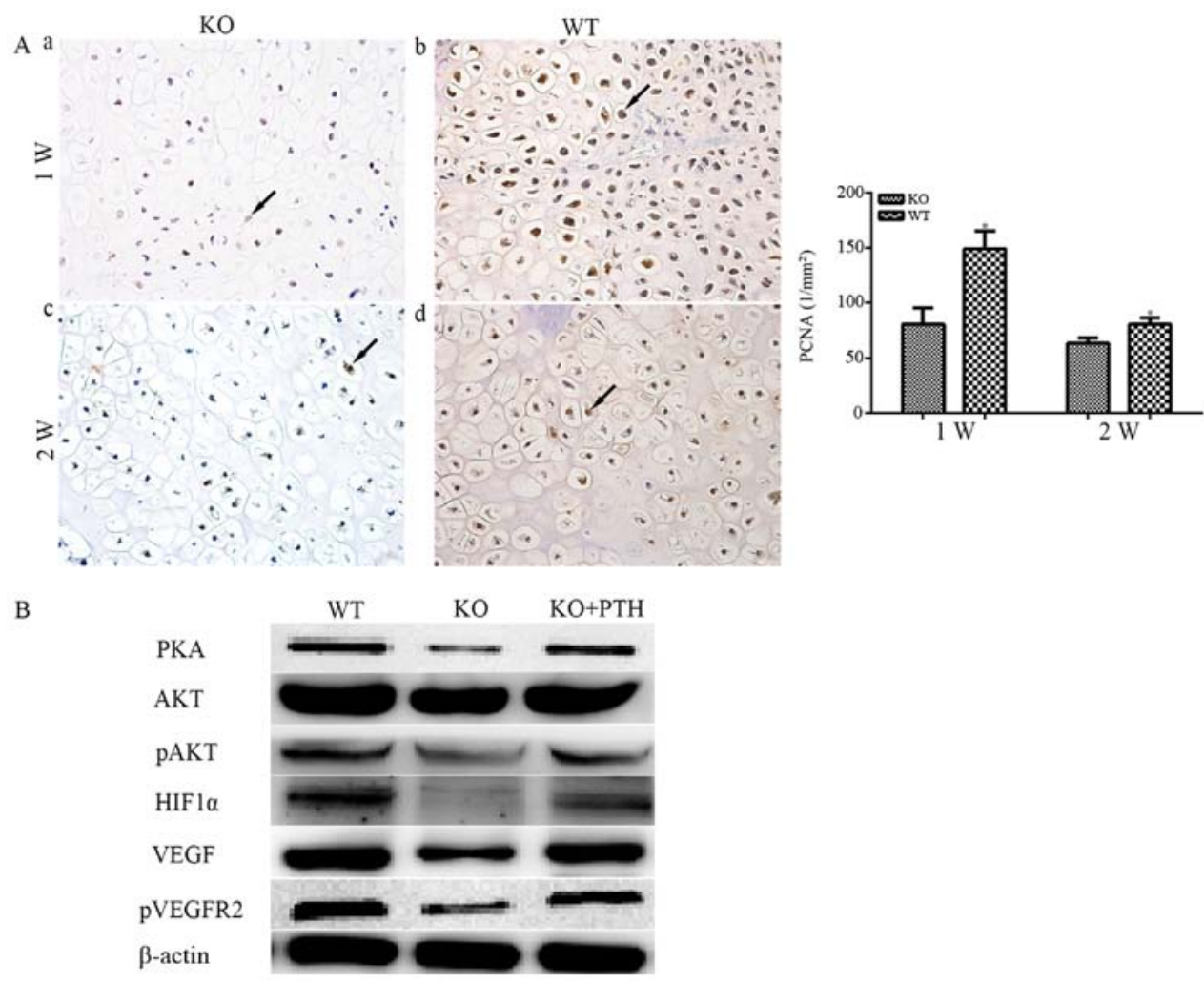

Figure 6. (A) Immunohistochemical staining of PCNA (magnification, x400) in fractures. (a) In PTHKO and (b) WT mice 1 week after fracture, rapid cell proliferation was observed, whereas PTHKO mice exhibited a significant reduction in PCNA-positive rate compared with in WT mice. (c) In PTHKO and (d) WT mice 2 weeks after fracture, cell proliferation in WT mice remained faster than that in PTHKO mice; however, it slowed down in both groups. Black arrows indicate positive areas. ${ }^{*} \mathrm{P}<0.05$. (B) Western blot analysis detected reduced expression of PKA/pAKT/HIF1 $\alpha /$ VEGF, and a lower level of pVEGFR2 in PTHKO BMSC-derived osteoblasts 2 weeks after induction. Addition of exogenous PTH in the culture medium partially reversed downregulation of the PKA/pAKT/HIF1 $\alpha /$ VEGF pathway. AKT, serine/threonine protein kinase; BMSC, bone marrow mesenchymal stem cell; HIF1 $\alpha$, hypoxia inducible factor-1 $\alpha$; pAKT, phosphorylated-AKT; PCNA, proliferating cell nuclear antigen; PKA, protein kinase A; PTH, parathroid hormone; PTHKO, PTH knockout; pVEGFR2, p-VEGFR2; VEGF, vascular endothelial growth factor; VEGFR2, VEGF receptor 2; WT, wild-type.

in WT mice, and was concentrated in areas adjacent to the cartilage callus (Fig. 3Bc and d).

Callus-specific proteins, including OCN and RUNX2, were semi-quantified by western blot analysis. The results were consistent with those of immunohistochemical staining (Fig. 3C).

BMSCs were isolated from mouse femoral bone marrow, and osteoblastic differentiation was induced with dexamethasone, ascorbic acid and $\beta$-glycerophosphate, in order to investigate whether lack of endogenous PTH affects differentiation of mesenchymal stem cells into osteoblasts. Immunocytochemical analysis revealed markedly lower levels of ALP in PTHKO mice compared with in WT mice, thus indicating a diminished capacity of BMSCs for osteoblastic differentiation in PTHKO mice (Fig. 3D). Total RNA was extracted 7 and 14 days after cell culture, and osteogenic indicators were quantified by RT-qPCR. The results indicated that the mRNA expression levels of osteoblast-specific proteins, including RUNX2 and OCN, were significantly lower in PTHKO mice compared with in WT mice (Fig. 3Ea and b).

Reduced angiogenesis in PTHKO mice. Fracture healing involves the remodeling of blood vessels (3), and endochondral bone formation is closely associated with angiogenesis. The present study hypothesized that defective endochondral bone formation in PTHKO mice may be associated with decreased angiogenic capacity. Immunohistochemical detection of the vascular specific marker PECAM confirmed this hypothesis. The area of angiogenesis around the edge of the callus in WT mice was significantly larger than that in PTHKO mice (Fig. 4Aa and b) 1 week after fracture. In addition, a large number of blood vessels were observed in the cartilage callus in WT mice 2 weeks after fracture, whereas PTHKO mice exhibited far fewer blood vessels, which were mainly located around the cartilage callus (Fig. 4Ac and d).

BMSC-derived osteoblasts and HUVECs were cocultured for 2 weeks, in order to confirm the adverse effects of the impaired osteoblastic differentiation capacity of BMSCs and their decreased angiogenic activity. The results indicated that HUVECs cocultured with BMSCs from PTHKO mice exhibited significantly reduced capacity for aggregation and cross-linking (Fig. 4B).

Various growth factors are required for angiogenesis in the process of fracture healing, and VEGF is particularly important $(29,30)$. Immunohistochemical analysis demonstrated that the expression of VEGF-A was significantly increased in WT mice compared with in PTHKO mice (Fig. 5Aa and b) 1 week after fracture. VEGF-A expression remained higher than that in WT mice 2 weeks after fracture (Fig. 5Ac and d) even though it was slightly increased in WT and PTHKO mice compared with at 1 week. The receptor tyrosine kinase VEGFR2 is phosphorylated and activated by binding to extracellular 
VEGF-A and mediates almost all known VEGF-A-induced cellular responses (31). Immunohistochemical analysis detected pVEGFR2 in a large number of callus cells in WT mice 1 week after fracture (Fig. 5Bb), whereas far fewer positive cells were detected in PTHKO mice (Fig. 5Ba). In addition, PTHKO mice possessed significantly fewer pVEGFR2-positive cells in the cartilage callus compared with in WT mice 2 weeks after fracture (Fig. 5Bc and d). HIF1 $\alpha$ expression within the callus was examined to determine the association between HIF1 $\alpha$ and VEGF (30). Whereas strong cytoplasmic and endonulcear HIF1 $\alpha$ expression was observed in WT mice 1 week after fracture, HIF1 $\alpha$ expression was much weaker in PTHKO mice (Fig. 5Ca and b). HIF1 $\alpha$ expression increased more slowly in PTHKO mice compared with in WT mice; however, stronger expression was detected in both mice groups 2 weeks after fracture (Fig. 5Cc and d). In addition, the protein expression levels of VEGF, pVEGFR2 and HIF1 $\alpha$ were determined by western blot analysis. The results were similar to those of immunohistochemical staining (Fig. 5D).

Downregulation of cell proliferation and the PKA/pAKT/HIF1 $/$ /VEGF signaling pathway in PTHKO mice. It has previously been reported that exogenous $\mathrm{PTH}$ can reduce DNA damage caused by oxidative stress (32); however, the relationship between endogenous PTH and the local oxygen environment, as well as its association with cell proliferation, remain to be elucidated. Since endogenous PTH can affect angiogenesis and consequently affect the oxygen supply during fracture healing, it may also influence cell proliferation. PCNA was measured in PTHKO and WT mice, and rapid cell proliferation was observed in both groups 1 week after fracture. However, the PCNA-positive rate was significantly reduced in PTHKO mice compared with in WT mice (Fig. 6Aa and b). Cell proliferation was reduced in both groups 2 weeks after fracture, and the PCNA-positive rate in PTHKO mice remained lower than that in WT mice (Fig. 6Ac and d), thus suggesting that lack of endogenous PTH may cause reduced callus formation by suppressing cell proliferation.

Previous studies have confirmed that exogenous PTH can promote VEGF secretion from osteoblasts $(13,26)$. BMSCs were isolated from mouse femoral bone marrow, and osteoblastic differentiation was induced with dexamethasone, ascorbic acid and $\beta$-glycerophosphate to investigate whether lack of endogenous PTH affects the VEGF pathway. Western blot analysis revealed that the expression of proteins involved in the PKA/pAKT/HIF1 $\alpha$ signaling pathway were reduced 2 weeks after induction. In addition, a reduction in VEGF and pVEGFR2 expression was observed. Conversely, administration of exogenous PTH to cells from PTHKO mice partially reversed downregulation of the PKA/pAKT/HIF1 $\alpha / \mathrm{VEGF}$ pathway (Fig. 6B).

\section{Discussion}

In the present study, PTHKO mice displayed delayed fracture healing alongside formation of a smaller callus, defective endochondral bone formation and reduced osteoblast activity. Angiogenic markers were measured, due to the close association between angiogenesis and endochondral bone formation (27). Decreased expression of VEGF and the oxygen sensor HIF1 $\alpha$ was observed in PTHKO mice, whereas reduced angiogenesis was associated with endochondral bone formation. In vitro experiments confirmed that lack of endogenous PTH inhibited osteoblastic differentiation of mesenchymal stem cells and downregulated signal transduction of the PKA/pAKT/HIF1 $\alpha /$ VEGF pathway. In addition, the aggregation and crosslinking of endothelial cells was reduced when cocultured with BMSCs from PTHKO mice. Furthermore, in vitro experiments demonstrated that exogenous PTH partially reversed reduced signal transduction of the PKA/pAKT/HIF1 $\alpha /$ VEGF pathway.

The regeneration of blood vessels is crucial for callus remodeling during fracture healing (3). In the early stage of fracture healing, low oxygen levels induce a large number of differentiated chondrocytes to form a cartilage callus. As mature chondrocytes begin to secrete cartilage matrix factors, blood vessels grow into the cartilage callus along the matrix, resulting in increased levels of oxygen, which induce osteoblastic differentiation and gradual mineralization of the matrix, thus resulting in the generation of a large amount of woven bone (3-5). Therefore, angiogenesis is closely associated with endochondral bone formation. PTH has an anabolic effect on bone; however, its association with angiogenesis remains unclear. The present study demonstrated that lack of endogenous PTH may cause a reduction in angiogenesis and defects in endochondral bone formation, ultimately leading to delayed fracture healing.

Hypoxia is a major regulatory factor during angiogenesis. The HIF signaling pathway is the key pathway by which most body tissues react to local partial pressures of oxygen, and HIF is simultaneously influenced by various hormones, as well as nerve activity $(6,22)$. Li et al reported that the osteogenic effects of PTH are predominantly mediated by the AC-cAMP-PKA pathway (21). Park et al also indicated that, in tumor cells, activation of the $\beta$-adrenergic receptor promotes angiogenesis by upregulating VEGF expression via the cAMP/ PKA/phosphatidylinositol 3-kinase/Akt/mammalian target of rapamycin/p70S6 kinase/HIF1 $\alpha /$ VEGF pathway (22). The present study confirmed that the expression levels of all proteins involved in the PKA/pAKT/HIF1 $\alpha$ pathway were reduced in PTHKO mouse BMSC-derived osteoblasts, whereas exogenous PTH partially increased their expression. These findings suggested that PTH may affect the expression of VEGF via the PKA/pAKT/HIF1 $\alpha$ pathway and thereby influence angiogenesis.

At present, VEGF is the most well-studied angiogenic factor in the mammalian skeletal system. As the most important HIF1 $\alpha$ target gene following its translocation into the nucleus, VEGF is considered the crucial link between angiogenesis and osteogenesis $(9,10,12,27)$. Numerous studies have demonstrated that increased VEGF expression in osteoblasts may be induced by various stimuli $(20,23)$, and that VEGF can increase the activities of endothelial cells and promote angiogenesis $(33,34)$. In addition, it has been reported that VEGF can act directly on osteogenic progenitor cells, thus enhancing their recruitment and differentiation, and promoting fracture healing $(33,35)$. Deletion of the endogenous VEGF gene in mice leads to impaired BMSC-derived osteoblast functions; this finding confirms that VEGF may directly affect differen- 
tiation, maturation and functionality of osteoblasts (12). The present study revealed that the expression levels of VEGF were reduced, and that osteoblast activity and angiogenesis were inhibited in PTHKO mice. However, further studies are required to elucidate whether VEGF directly affects the functions of osteoblasts or acts through its effects on angiogenesis. The present in vitro experiments detected lower VEGF expression in PTHKO mouse BMSC-derived osteoblasts compared with in those from WT mice. In addition, osteoblasts from PTHKO mice exhibited a diminished ability to promote aggregation and cross-linking of endothelial cells in a coculture experiment. These findings also indicated that these cells exhibited reduced ability to promote angiogenesis. Therefore, it may be hypothesized that it is more likely that endogenous PTH affects fracture healing indirectly by regulating angiogenesis via the PKA/pAKT/HIF1 $\alpha /$ VEGF pathway.

Mature cartilage cells can continuously secrete cartilage matrix factors, which provide a scaffold for vascular invasion into the callus (3-5). Previous studies have suggested that mature osteoclasts can produce heparanase during the fracture healing process, which degrades proteoglycans containing sulfated heparin in the extracellular matrix and results in the release of heparin-associated growth factors, including VEGF and basic fibroblast growth factor $(36,37)$. These factors can increase angiogenesis, and regulate the activities of osteoblasts and osteoclasts. The present study demonstrated that the expression of matrix proteins, including COL II, OCN and ALP were decreased, and VEGF expression was also reduced, thus suggesting that the lack of endogenous PTH may induce a simultaneous decrease in bone matrix and angiogenesis. However, further research is required to clarify the interaction between these two processes.

In conclusion, the present study suggested that the lack of endogenous PTH may decrease expression of VEGF in BMSCs or osteoblasts via downregulation of the $\mathrm{PKA} / \mathrm{pAKT} / \mathrm{HIF} 1 \alpha / \mathrm{VEGF}$ pathway, which causes reduced angiogenesis and thereby affects endochondral bone formation, ultimately leading to delayed fracture healing.

\section{Acknowledgements}

The present study was supported by the National Natural and Science Foundation of China (grant nos. 81472080 and 81520108018).

\section{Competing interests}

The authors declare that they have no competing interests.

\section{References}

1. Gerstenfeld LC, Cullinane DM, Barnes GL, Graves DT and Einhorn TA: Fracture healing as a post-natal developmental process: Molecular, spatial, and temporal aspects of its regulation. J Cell Biochem 88: 873-884, 2003.

2. Tsiridis E, Upadhyay N and Giannoudis P: Molecular aspects of fracture healing: Which are the important molecules? Injury 38 (Suppl 1): S11-S25, 2007.

3. Geris L, Gerisch A, Sloten JV, Weiner R and Oosterwyck HV: Angiogenesis in bone fracture healing: A bioregulatory model. J Theor Biol 251: 137-158, 2008.

4. Taguchi K, Ogawa R, Migita M, Hanawa $\mathrm{H}$, Ito $\mathrm{H}$ and Orimo $\mathrm{H}$ : The role of bone marrow-derived cells in bone fracture repair in a green fluorescent protein chimeric mouse model. Biochem Biophys Res Commun 331: 31-36, 2005 .
5. Khosla S, Westendorf JJ, Oursler MJ. Building bone to reverse osteoporosis and repair fractures. The Journal of Clinical lnvestigation 118: 421-428, 2008. 10.1172/JCI33612.

6. Wan C, Gilbert SR, Wang Y, Cao X, Shen X, Ramaswamy G, Jacobsen KA, Alaql ZS, Eberhardt AW, Gerstenfeld LC, et al: Activation of the hypoxia-inducible factor-1alpha pathway accelerates bone regeneration. Proc Natl Acad Sci USA 105: 686-691, 2008.

7. Ivan M, Kondo K, Yang H, Kim W, Valiando J, Ohh M, Salic A, Asara JM, Lane WS and Kaelin WG Jr: HIFalpha targeted for VHL-mediated destruction by proline hydroxylation: Implications for O2 sensing. Science 292: 464-468, 2001.

8. Jaakkola P, Mole DR, Tian YM, Wilson MI, Gielbert J, Gaskell SJ, von Kriegsheim A, Hebestreit HF, Mukherji M, Schofield CJ, et al: Targeting of HIF-alpha to the von Hippel-Lindau ubiquitylation complex by O2-regulated prolyl hydroxylation. Science 292: 468-472, 2001.

9. Kallio PJ, Okamoto K, O'Brien S, Carrero P, Makino Y, Tanaka H and Poellinger L: Signal transduction in hypoxic cells: Inducible nuclear translocation and recruitment of the $\mathrm{CBP} / \mathrm{p} 300$ coactivator by the hypoxia-inducible factor-1alpha. EMBO J 17: 6573-6586, 1998.

10. Kusumbe AP, Ramasamy SK and Adams RH: Coupling of angiogenesis and osteogenesis by a specific vessel subtype in bone. Nature 507: 323-328, 2014.

11. Brahimi-Horn MC and Pouysségur J: Harnessing the hypoxia-inducible factor in cancer and ischemic disease. Biochem Pharmacol 73: 450-457, 2007.

12. Liu Y, Berendsen AD, Jia S, Lotinun S, Baron R, Ferrara N and Olsen BR: Intracellular VEGF regulates the balance between osteoblast and adipocyte differentiation. J Clin Invest 122: 3101-3113, 2012.

13. Gensure RC, Gardella TJ and Jüppner H: Parathyroid hormone and parathyroid hormone-related peptide, and their receptors. Biochem Biophys Res Commun 328: 666-678, 2005.

14. Barnes GL, Kakar S, Vora S, Morgan EF, Gerstenfeld LC and Einhorn TA: Stimulation of fracture-healing with systemic intermittent parathyroid hormone treatment. J Bone Joint Surg Am 90 (Suppl 1): 120-127, 2008.

15. Miao D, He B, Karaplis AC and Goltzman D: Parathyroid hormone is essential for normal fetal bone formation. J Clin Invest 109: 1173-1182, 2002

16. Xue Y, Karaplis AC, Hendy GN, Goltzman D and Miao D: Genetic models show that parathyroid hormone and 1,25-dihydroxyvitamin D3 play distinct and synergistic roles in postnatal mineral ion homeostasis and skeletal development. Hum Mol Genet 14: 1515-1528, 2005.

17. Ren Y, Liu B, Feng Y, Shu L, Cao X, Karaplis A, Goltzman D and Miao D: Endogenous PTH deficiency impairs fracture healing and impedes the fracture-healing efficacy of exogenous PTH(1-34). PLoS One 6: e23060, 2011.

18. Jilka RL, O'Brien CA, Bartell SM, Weinstein RS and Manolagas SC: Continuous elevation of PTH increases the number of osteoblasts via both osteoclast-dependent and -independent mechanisms. J Bone Miner Res 25: 2427-2437, 2010.

19. Rhee Y, Park SY, Kim YM, Lee S and Lim SK: Angiogenesis inhibitor attenuates parathyroid hormone-induced anabolic effect. Biomed Pharmacother 63: 63-68, 2009.

20. Prisby R, Guignandon A, Vanden-Bossche A, Mac-Way F, Linossier MT, Thomas M, Laroche N, Malaval L, Langer M, Peter ZA, et al: Intermittent PTH(1-84) is osteoanabolic but not osteoangiogenic and relocates bone marrow blood vessels closer to bone-forming sites. J Bone Miner Res 26: 2583-2596, 2011.

21. Li X, Liu H, Qin L, Tamasi J, Bergenstock M, Shapses S, Feyen JH, Notterman DA and Partridge NC: Determination of dual effects of parathyroid hormone on skeletal gene expression in vivo by microarray and network analysis. J Biol Chem 282: 33086-33097, 2007.

22. Park SY, Kang JH, Jeong KJ, Lee J, Han JW, Choi WS, Kim YK, Kang J, Park CG and Lee HY: Norepinephrine induces VEGF expression and angiogenesis by a hypoxia-inducible factor- $1 \alpha$ protein-dependent mechanism. Int J Cancer 128: 2306-2316, 2011.

23. Esbrit P, Alvarez-Arroyo MV, De Miguel F, Martin O, Martinez ME and Caramelo C: C-terminal parathyroid hormone-related protein increases vascular endothelial growth factor in human osteoblastic cells. J Am Soc Nephrol 11: 1085-1092, 2000. 
24. Miao D, He B, Lanske B, Bai XY, Tong XK, Hendy GN, Goltzman D and Karaplis AC: Skeletal abnormalities in Pth-null mice are influenced by dietary calcium. Endocrinology 145: 2046-2053, 2004.

25. Marturano JE, Cleveland BC, Byrne MA, O'Connell SL, Wixted JJ and Billiar KL: An improved murine femur fracture device for bone healing studies. J Biomech 41: 1222-1228, 2008

26. Menon P, Yin G, Smolock EM, Zuscik MJ, Yan C and Berk BC: GPCR kinase 2 interacting protein 1 (GIT1) regulates osteoclast function and bone mass. J Cell Physiol 225: 777-785, 2010.

27. Yin G, Sheu TJ, Menon P, Pang J, Ho HC, Shi S, Xie C, Smolock E, Yan C, Zuscik MJ, et al: Impaired angiogenesis during fracture healing in GPCR kinase 2 interacting protein-1 (GIT1) knock out mice. PLoS One 9: e89127, 2014.

28. Krishnan V, Moore TL, Ma YL, Helvering LM, Frolik CA, Valasek KM, Ducy P and Geiser AG: Parathyroid hormone bone anabolic action requires Cbfa1/Runx2-dependent signaling. Mol Endocrinol 17: 423-435, 2003.

29. Olsson AK, Dimberg A, Kreuger J and Claesson-Welsh L: VEGF receptor signalling - in control of vascular function. Nat Rev Mol Cell Biol 7: 359-371, 2006.

30. Tischer E, Mitchell R, Hartman T, Silva M, Gospodarowicz D, Fiddes JC and Abraham JA: The human gene for vascular endothelial growth factor. Multiple protein forms are encoded through alternative exon splicing. J Biol Chem 266 11947-11954, 1991.

31. Holmes K, Roberts OL, Thomas AM and Cross MJ: Vascular endothelial growth factor receptor-2: Structure, function, intracellular signalling and therapeutic inhibition. Cell Signal 19: 2003-2012, 2007.

32. Schnoke M, Midura SB and Midura RJ: Parathyroid hormone suppresses osteoblast apoptosis by augmenting DNA repair. Bone 45: 590-602, 2009.
33. Mayr-Wohlfart U, Waltenberger J, Hausser H, Kessler S, Günther KP, Dehio C, Puhl W and Brenner RE: Vascular endothelial growth factor stimulates chemotactic migration of primary human osteoblasts. Bone 30: 472-477, 2002.

34. Street J, Bao M, deGuzman L, Bunting S, Peale FV Jr, Ferrara N, Steinmetz H, Hoeffel J, Cleland JL, Daugherty A, et al: Vascular endothelial growth factor stimulates bone repair by promoting angiogenesis and bone turnover. Proc Natl Acad Sci USA 99: 9656-9661, 2002.

35. Keramaris NC, Calori GM, Nikolaou VS, Schemitsch EH and Giannoudis PV: Fracture vascularity and bone healing: A systematic review of the role of VEGF. Injury 39 (Suppl 2): S45-S57, 2008

36. Collin-Osdoby P, Rothe L, Bekker S, Anderson F, Huang Y and Osdoby P: Basic fibroblast growth factor stimulates osteoclast recruitment, development, and bone pit resorption in association with angiogenesis in vivo on the chick chorioallantoic membrane and activates isolated avian osteoclast resorption in vitro. J Bone Miner Res 17: 1859-1871, 2002.

37. Saijo M, Kitazawa R, Nakajima M, Kurosaka M, Maeda S and Kitazawa S: Heparanase mRNA expression during fracture repair in mice. Histochem Cell Biol 120: 493-503, 2003.

This work is licensed under a Creative Commons Attribution-NonCommercial-NoDerivatives 4.0 International (CC BY-NC-ND 4.0) License. 